\title{
Surface Functionalization of Reconstructed Si(111)
}

\section{with Methionine}

Fatemeh Rahnemaye Rahsepar ${ }^{1,2 *}$ and Kam Tong Leung ${ }^{2 *}$

${ }^{1}$ School of Chemistry, College of Science, University of Tehran, Tehran, Iran

${ }^{2}$ WATLab and Department of Chemistry, University of Waterloo, Waterloo, Ontario N2L 3G1, Canada

Corresponding Authors

*Email: tong@uwaterloo.ca

frahsepar@ut.ac.ir 

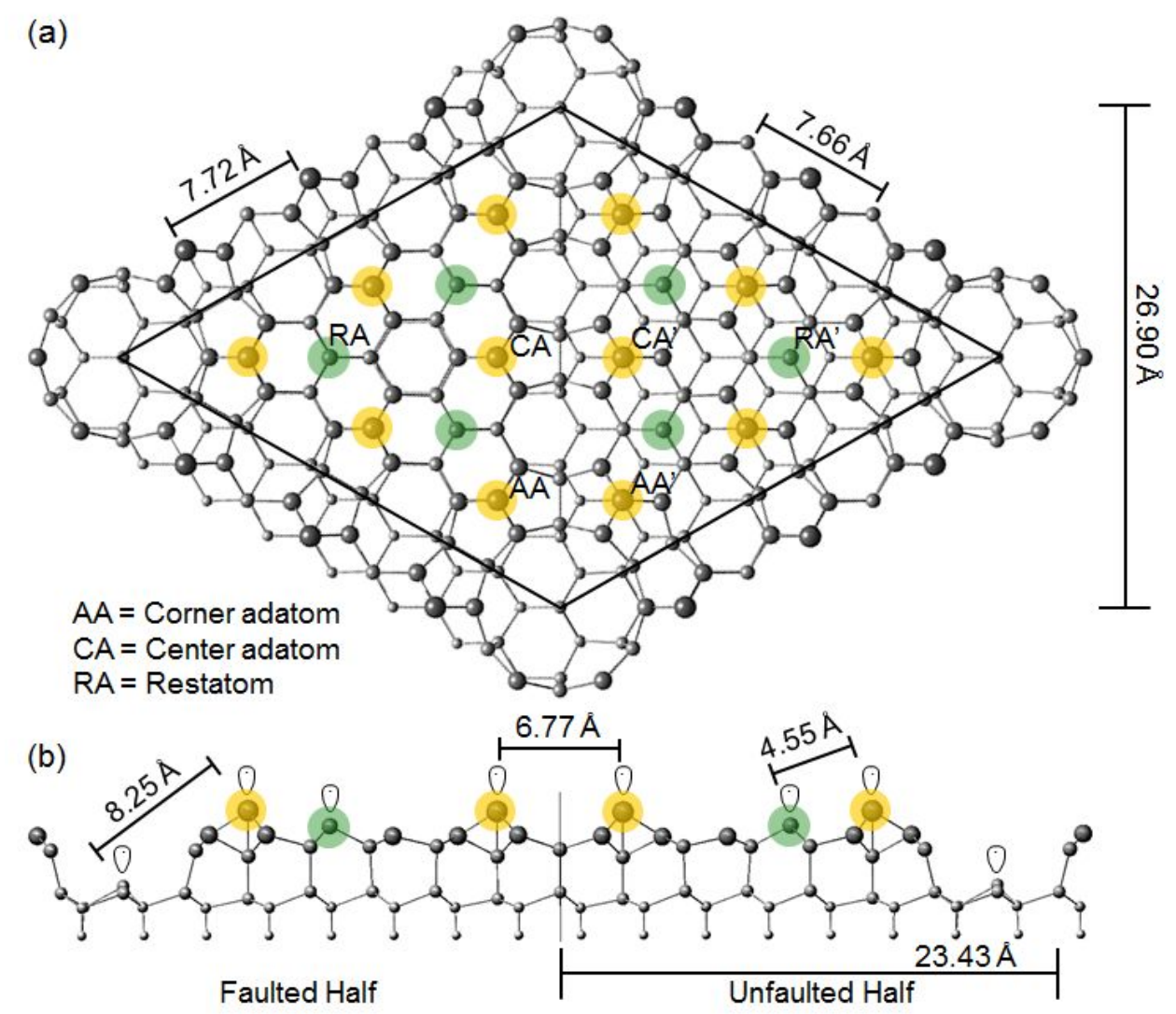

Figure S1. (a) Top and (b) side views of the equilibrium dimer-adatom-stacking fault (DAS) model of a $\mathrm{Si}_{200} \mathrm{H}_{49}$ cluster (consisting of a top Si adatom layer, two Si bilayers, and a bottom layer of terminating $\mathrm{H}$ atoms) obtained by large-scale DFT-D2 calculations used as the model surface for $\operatorname{Si}(111) 7 \times 7$. Spheres with decreasing sizes are used to represent the Si atoms with increasing distance from the surface. Dangling bonds are identified on the top of the adatoms, restatoms, and corner holes. For clarity, silicon adatoms and restatoms are highlighted, respectively, by larger yellow and green circles. 
Table S1. Binding energies (in eV) of fitted peak maxima for various core-level features and their assignments for different exposures of L-methionine on $\mathrm{Si}(111) 7 \times 7$ and for $\mathrm{L}$-methionine powder.

\begin{tabular}{|c|c|c|c|c|c|c|c|c|c|}
\hline \multirow{2}{*}{$\begin{array}{l}\text { Core } \\
\text { level }\end{array}$} & \multirow{2}{*}{\begin{tabular}{|c|}
$\begin{array}{c}\text { Interfacial } \\
\text { layer }\end{array}$ \\
$30 \mathrm{~s}$ \\
\end{tabular}} & \multicolumn{4}{|c|}{ Transitional layer } & \multicolumn{2}{|c|}{ Multilayer } & \multirow{2}{*}{ Powder } & \multirow{2}{*}{ Assignment $t^{\#}$} \\
\hline & & $90 \mathrm{~s}$ & $180 \mathrm{~s}$ & $270 \mathrm{~s}$ & $540 \mathrm{~s}$ & $1080 \mathrm{~s}$ & $2160 s$ & & \\
\hline \multirow{2}{*}{ O 1s } & 532.5 & 532.6 & 532.5 & 532.4 & 532.2 & & & & $-\mathrm{OH} /-\mathrm{C}=\mathrm{O}$ \\
\hline & & & 531.9 & 531.8 & 531.8 & 531.9 & 531.8 & 531.9 & $-\mathrm{COO}^{-}$ \\
\hline \multirow{3}{*}{ N 1s } & 398.7 & 398.7 & 398.7 & 398.7 & 398.7 & & & & $-\mathrm{NH}-\mathrm{Si}$ \\
\hline & & 401.0 & 401.0 & 401.0 & 401.1 & & & & $\mathrm{O}-\mathrm{H} \cdots \mathrm{N}$ \\
\hline & & & 401.8 & 401.8 & 401.8 & 401.8 & 401.8 & 401.8 & $-\mathrm{NH}_{3}^{+}$ \\
\hline \multirow{5}{*}{ C 1s } & 285.0 & 285.1 & 285.4 & 285.2 & 285.3 & 285.4 & 285.4 & 285.5 & $-\mathrm{CH}_{2}-$ \\
\hline & 285.7 & 285.8 & 285.9 & 285.8 & 285.9 & 285.9 & 285.9 & 286.0 & $-\mathrm{CH}_{2}-\mathrm{S}-\mathrm{CH}_{3}$ \\
\hline & 286.5 & 286.6 & 286.8 & & & & & & $-\mathrm{CH}-\mathrm{NH}-\mathrm{Si}$ \\
\hline & & & & 286.6 & 286.7 & 286.8 & 286.8 & 286.9 & $-\mathrm{CH}-\mathrm{NH}_{3}{ }^{+}$ \\
\hline & 288.9 & 289.3 & 288.9 & 288.8 & 288.9 & 288.9 & 288.8 & 288.9 & $\begin{array}{l}-\mathrm{COOH} \\
-\mathrm{COO}^{-}\end{array}$ \\
\hline S 2s & & 228.1 & 228.1 & 228.1 & 228.1 & 228.1 & 228.1 & & $-\mathrm{CH}_{2}-\mathrm{S}-\mathrm{CH}_{3}$ \\
\hline
\end{tabular}

\# Triple-dot line “..." is used to represent a hydrogen bond. 
Table S2. Bond lengths, bond angles, and dihedral angles of equilibrium structures of (c1) free L-methionine and the most stable adsorption configurations of L-methionine (c2) across the dimer wall and (c3) within a half unit cell of the $\operatorname{Si}(111) 7 \times 7$.

\begin{tabular}{|c|c|c|c|c|c|c|c|c|c|c|c|}
\hline $\begin{array}{c}\text { Bond } \\
\text { length }(\AA) \\
\end{array}$ & C1 & $\mathrm{C} 2$ & C3 & $\begin{array}{c}\text { Bond angle } \\
\left({ }^{\circ}\right)\end{array}$ & C1 & $\mathrm{C2}$ & $\mathrm{C} 3$ & $\begin{array}{l}\text { Dihedral } \\
\text { angle }\left({ }^{\circ}\right)\end{array}$ & C1 & $\mathrm{C} 2$ & $\mathrm{C3}$ \\
\hline $\mathrm{C} 1-\mathrm{O} 1$ & 1.3647 & 1.3662 & 1.3182 & $\mathrm{O} 1-\mathrm{C} 1-\mathrm{O} 2$ & 122.93 & 123.09 & 124.98 & $\begin{array}{l}\mathrm{H} 1-\mathrm{O} 1-\mathrm{C} 1- \\
\mathrm{O} 2\end{array}$ & -1.45 & -2.20 & 3.90 \\
\hline $\mathrm{C} 1=\mathrm{O} 2$ & 1.2199 & 1.2175 & 1.2409 & $\mathrm{O} 2-\mathrm{C} 1-\mathrm{C} 2$ & 125.09 & 124.55 & 121.72 & $\mathrm{O} 2-\mathrm{C} 1-\mathrm{C} 2-\mathrm{N}$ & -22.30 & 20.10 & -4.85 \\
\hline $\mathrm{C} 1-\mathrm{C} 2$ & 1.5287 & 1.5452 & 1.5352 & $\mathrm{O} 1-\mathrm{C} 1-\mathrm{C} 2$ & 111.97 & 112.32 & 113.29 & $\mathrm{O} 1-\mathrm{C} 1-\mathrm{C} 2-\mathrm{C} 3$ & -78.10 & -32.10 & -58.65 \\
\hline $\mathrm{C} 2-\mathrm{N}$ & 1.4567 & 1.4572 & 1.5416 & $\mathrm{C} 1-\mathrm{C} 2-\mathrm{N}$ & 113.31 & 111.81 & 113.15 & $\mathrm{O} 2-\mathrm{C} 1-\mathrm{C} 2-\mathrm{C} 3$ & 100.60 & 149.95 & 122.65 \\
\hline $\mathrm{C} 2-\mathrm{C} 3$ & 1.5437 & 1.5565 & 1.5488 & $\mathrm{C} 1-\mathrm{C} 2-\mathrm{C} 3$ & 108.18 & 111.79 & 109.36 & $\mathrm{~N}-\mathrm{C} 2-\mathrm{C} 3-\mathrm{C} 4$ & -63.05 & -46.50 & -47.05 \\
\hline $\mathrm{C} 3-\mathrm{C} 4$ & 1.5256 & 1.5319 & 1.5213 & $\mathrm{C} 2-\mathrm{C} 3-\mathrm{C} 4$ & 111.32 & 112.61 & 110.62 & $\mathrm{O} 1-\mathrm{C} 1-\mathrm{C} 2-\mathrm{N}$ & 169.75 & -158.05 & 173.90 \\
\hline C4-S & 1.8160 & 1.8371 & 1.8311 & C3-C4-S & 110.13 & 112.50 & 111.36 & $\mathrm{C} 1-\mathrm{C} 2-\mathrm{C} 3-\mathrm{C} 4$ & 175.40 & -168.95 & -168.40 \\
\hline S-C5 & 1.8080 & 1.8067 & 1.8064 & C4-S-C5 & 98.64 & 98.89 & 98.25 & C3-C4-S-C5 & -174.95 & 172.85 & 151.90 \\
\hline $\mathrm{N}-\mathrm{H} 3$ & 1.0233 & ------- & ------ & & & & & $\mathrm{H} 4-\mathrm{C} 5-\mathrm{S}-\mathrm{C} 4$ & -61.00 & -62.85 & -64.95 \\
\hline \multirow[t]{3}{*}{$\mathrm{N}-\mathrm{Si}$} & -------- & 1.7528 & 1.7455 & & & & & $\mathrm{C} 1-\mathrm{C} 2-\mathrm{N}-\mathrm{H} 2$ & -66.90 & -100.90 & -75.80 \\
\hline & & & & & & & & \begin{tabular}{|c|} 
H11-O1-C1- \\
C2
\end{tabular} & 177.30 & 179.85 & -174.80 \\
\hline & & & & & & & & C1-C2-N-H13 & 48.55 & ------ & -------- \\
\hline
\end{tabular}



(a) 0 1s $(\times 0.5)$
(b) N 1s
(c) C $1 \mathrm{~s}(\times 0.5)$
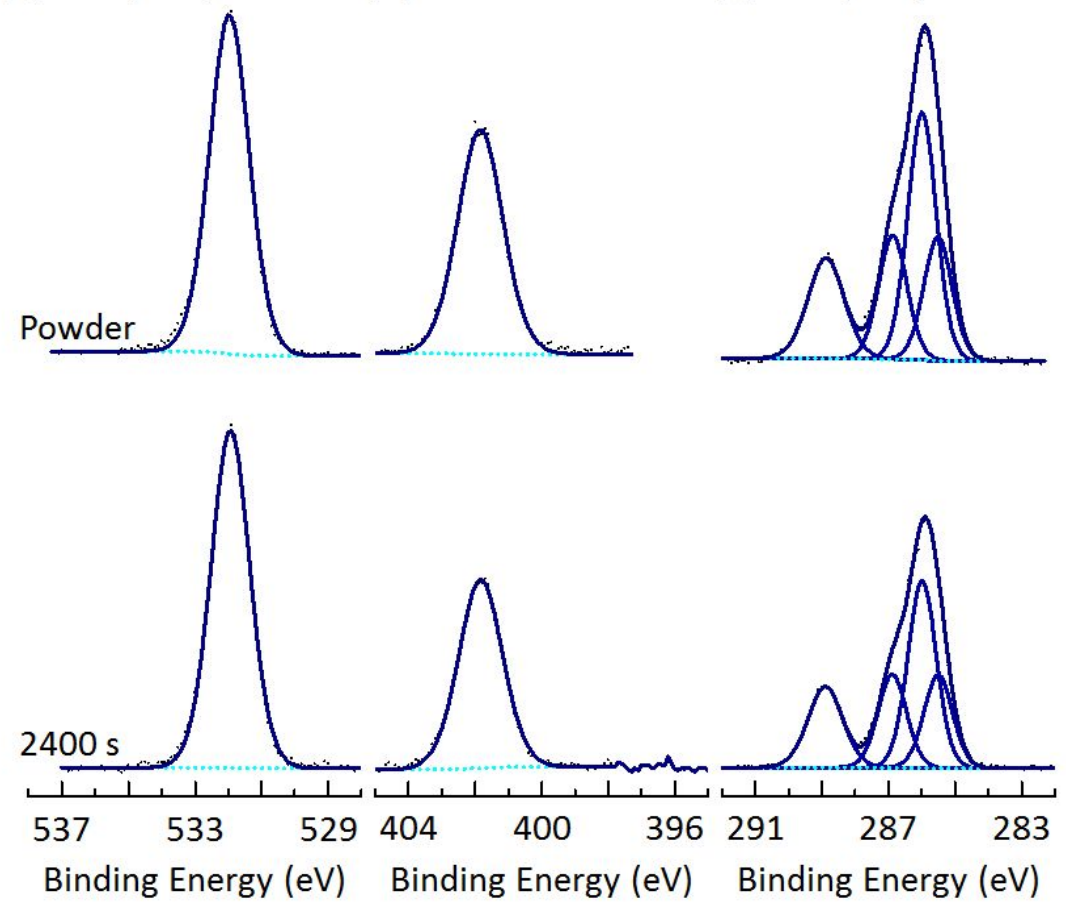

Figure S2. XPS spectra of (a) O 1s, (b) N 1s, and (c) C 1s regions for a methionine multilayer film (obtained with a $2400-\mathrm{s}$ exposure) on $\mathrm{Si}(111) 7 \times 7$ and for L-methionine powder. XPS data points are fitted with individual components (solid line) and a Shirley background (dotted line). 


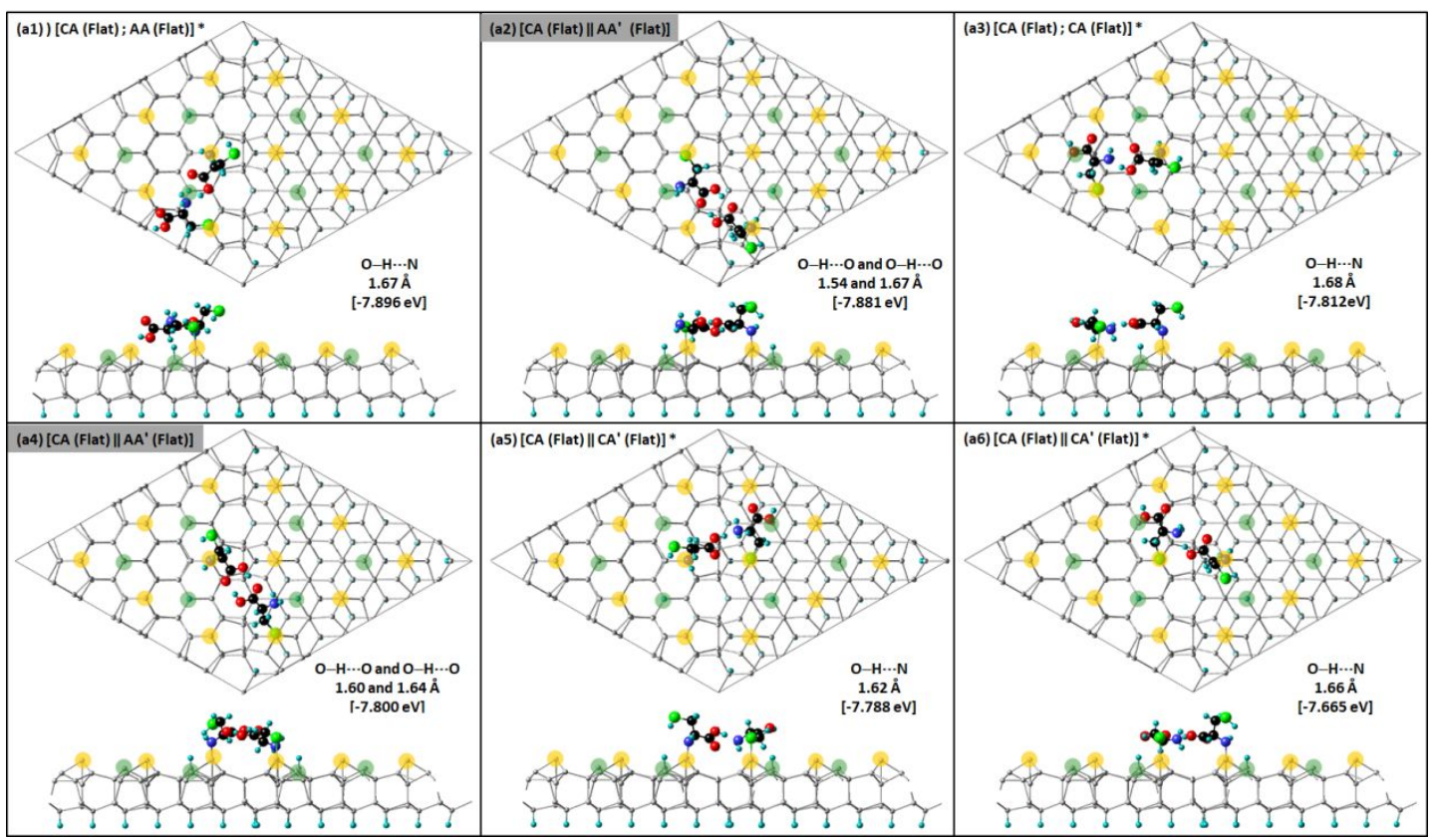




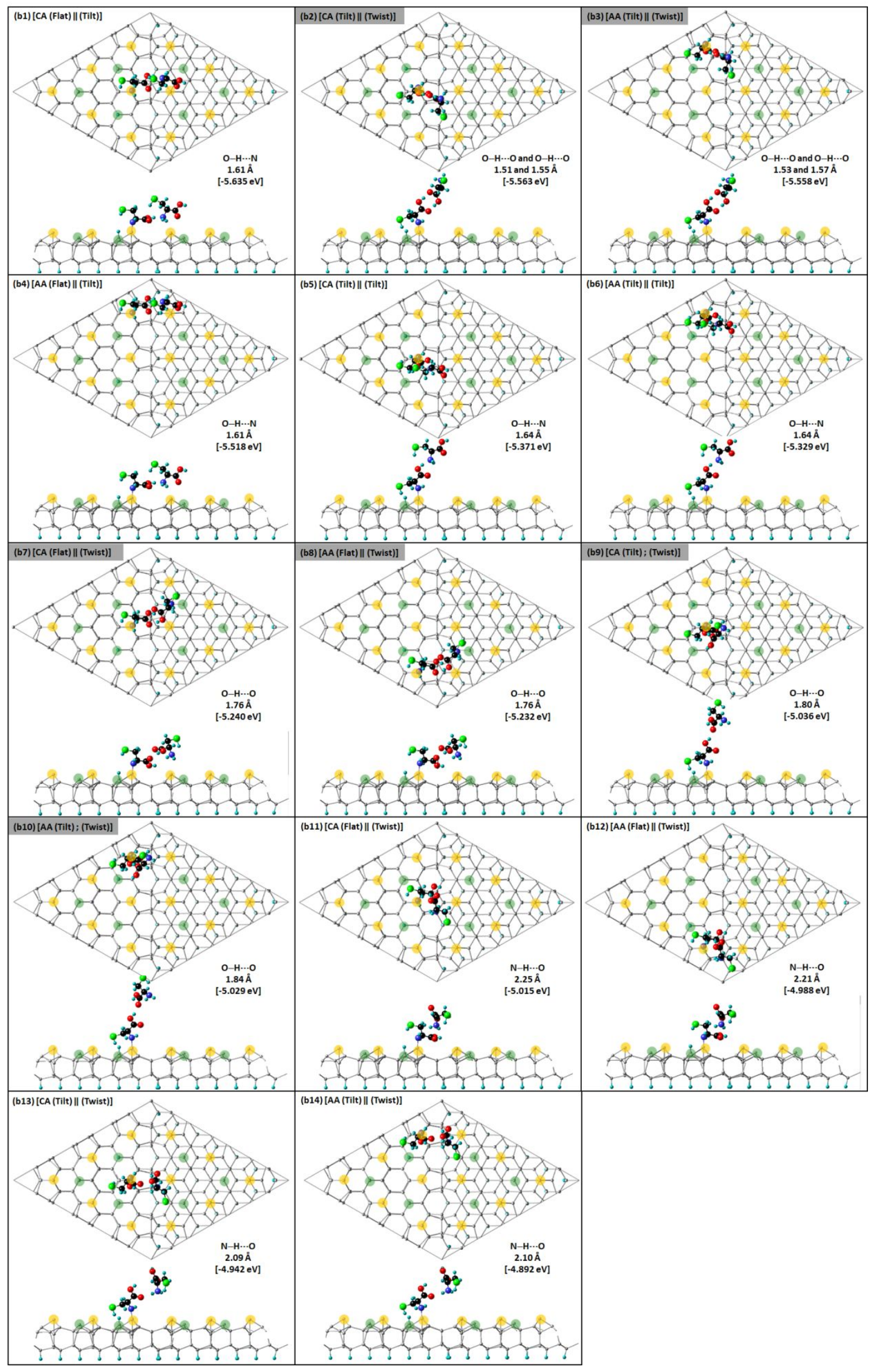


Figure S3. Top and side views of equilibrium di-molecular configurations of cysteine molecules for the most stable (a1-a6) "flat" and (b1-b14) "lateral" and "near-vertical" H-bonding on the $\mathrm{Si}_{200} \mathrm{H}_{49}$ model $7 \times 7$ surface, obtained by DFT-D2 calculations. The lengths of the respective $\mathrm{H}-$ bonds are indicated, along with the corresponding adsorption energies shown in square parentheses. Si adatoms and restatoms are highlighted, respectively, by larger yellow and green circles for clarity. Each panel heading describes the orientation of the $>\mathrm{C}-\mathrm{COOH}$ backbone in the admolecules with respect to the Si surface (flat, tilt, or twist) at specific Si adatom sites, with the double bar ( $\|$ ) and semicolon (;) indicating the second admolecule H-bonded, respectively, across the dimer wall and within the same half unit cell. The structures not supported by XPS results are grayed out, while plausible configurations (for early growth stage) supported by STM results are marked by asterisks. 